\title{
Rhetoric is Not the Answer
}

\author{
Allahbadia Gautam N.
}

Published online: 8 May 2014

(C) Federation of Obstetric \& Gynecological Societies of India 2014

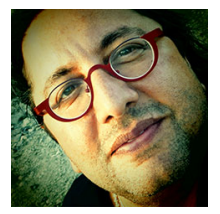

\section{About the Author}

Gautam N Allahbadia MD is the Editor-in-chief of the Journal of Obstetrics \& Gynecology of India as well as the IVF Lite (Journal of Minimal Stimulation IVF). He is the Medical Director of Rotunda -The Center for Human Reproduction, the world-renowned fertility clinic at Bandra, Mumbai, India as also the New Hope IVF Clinic at Sharjah, UAE. He is a noted world authority on Ultrasound guided Embryo Transfers and one of the pioneers in Third Party Reproduction in South-East Asia. Dr. Allahbadia was responsible for India's first trans-ethnic surrogate pregnancy involving a Chinese couple's baby delivered by an unrelated Indian surrogate mother. He has over 100 peer-reviewed publications to his credit and is on the Editorial Board of several International Journals. Throughout his career, Dr. Allahbadia has been instrumental in developing new fertility enhancing protocols and propagating the use of Ultrasound in Embryo Transfer procedures.

\section{Noun: Rhetoric}

The art of effective or persuasive speaking or writing, especially the exploitation of figures of speech and other compositional techniques.

"He is using a common figure of rhetoric, hyperbole"

Synonyms: oratory, eloquence, power of speech, command of language, expression, way with words, delivery, diction

Language designed to have a persuasive or impressive effect but which is often regarded as lacking in sincerity or meaningful content.

"All we have from the Opposition is empty rhetoric"

Synonyms: bombast, loftiness, turgidity, grandiloquence, magniloquence, ornateness, portentousness, pomposity, boastfulness, boasting, bragging, heroics, hyperbole, extravagant language, purple prose, pompousness, sonorousness

Allahbadia G. N. (\), Editor-in-chief

Rotunda-the Center For Human Reproduction, Bandra, Mumbai, India

e-mail: ivfwaladoc@gmail.com
Writing is hard. It is a very difficult process of intense concentration and brain work. As stated in Hayes' framework for the study of writing: "It is a generative activity requiring motivation, and it is an intellectual activity requiring cognitive processes and memory" [1]. In his book How to Write a Lot: A Practical Guide to Productive Academic Writing, Paul Silvia says that for some, "it's easier to embalm the dead than to write an article about it" [2].

Writing research papers does not come naturally to most of us. The typical research paper is a highly codified rhetorical form [3, 4]. Knowledge of the rules-some explicit, others implied-goes a long way toward writing a paper that will get accepted in a peer-reviewed journal. A good research paper addresses a specific research question. The research question-or study objective or main research hypothesis-is the central organizing principle of the paper. Whatever relates to the research question belongs in the paper; the rest does not. What is a good research question? The key attributes are (i) specificity; (ii) originality or novelty; and (iii) general relevance to a broad 
scientific community. The research question should be precise and not merely identify a general area of inquiry. Once the research question is clearly defined, writing the paper becomes considerably easier. The paper will ask the question, and then answer it.

The key to successful scientific writing is getting the structure of the paper right. Structure is the most difficult part of writing, no matter whether you are writing a novel, a play, a story, a research report, or a scientific paper. If the structure is right, then the rest can follow fairly easily, but no amount of clever language can compensate for a weak structure. Structure is important so that readers do not become lost. They should know where they have come from, where they are, and where they are headed. A strong structure also allows readers to know where to look for particular information and makes it more likely that all important information will be included.

The basic structure of a typical research paper is the sequence of Introduction, Methods, Results, and Discussion (sometimes abbreviated as IMRaD) [5]. Each section addresses a different objective. The authors state (i) the problem they intend to address-in other terms, the research question-in the Introduction; (ii) what they did to answer the question in the Methods section; (iii) what they observed in the Results section; and (iv) what they think the results mean in the Discussion. Readers have also become used to structured abstracts, which have been shown to include more important information than unstructured summaries [6].

Having the structure of the paper in place is a good start. However, there are many details that have to be attended to while writing. An obvious recommendation is to read and follow the Instructions to Authors published by the journal (typically the complete version is found on the Journal's website). Appropriate writing of the paper's title and the abstract is of utmost importance. Authors must realize that most readers read the title, some read the abstract, and only a few read the entire article. The title could be catchy to attract readers' attention, but it must be faithful to the research study. It cannot misrepresent the study. Writing a good abstract requires skill and experience. The abstract must be factual and goad the reader to read the whole article.

Visual elements are critical $[7,8]$. If readers go beyond the abstract, they are likely to examine the tables and figures next. Tables are typically used to display precise numeric values - a tool to make a paper more readable by removing numeric data from the text [9]. Tables can also be used to synthesize the existing literature, to explain variables, or to present the wording of survey questions.

A figure provides visual impact and thus is often the best way to communicate the primary finding. Clear, informative figures are invaluable; think creatively about how to use them. Work to develop a coherent set of visual elements that can stand alone-that is, tables and figures that not only convey the major result but also the basic methods.

A very important section of a paper is its reference list. In fact, it is the most important part of a paper according to me. Unfortunately, it is also the most neglected. It is mandatory that the authors must go through the references they are quoting - at least the relevant sections. Unfortunately, many authors merely include references quoted by other authors perhaps to impress the readers with their show of scholarship. This often results in incomplete and erroneous listing in the reference list. The unfortunate victim of this is the reader of your article who desires to go through a reference quoted by you but has to struggle to find it due to erroneous or incomplete listing. References quoted must be recent and relevant. There is really no justification for the author to quote any reference he has not bothered to read.

Presently, approximately $65 \%$ percent of the articles submitted to our Journal get rejected. The most important reasons for rejection are improper research methodology, poor study design, inadequate sample size, and absent or faulty statistical evaluation. To educate researchers, postgraduate students, their teachers and guides, readers of journals, and delegates attending conferences, the Journal started the Program for Inculcating the Culture of Scientific Enquiry and Pursuit (PICSEP) Project a few years back [10]. PICSEP spreads information, knowledge, and education regarding modern research methodology, basics of biostatistics, and science of writing a paper. The members on the Editorial Board of the Journal go as faculty members to speak on the subject of Research methodology to spread awareness on the Science of writing papers. To achieve its goals, PICSEP has developed many activities including

1) On site training of postgraduate students, their teachers $\&$ guides, and clinicians.

2) Projecting research methodology at YUVA Congresses, various other FOGSI-affiliated conferences, and CME programs.

3) Organizing PICSEP meetings.

4) The FOGSI-JOGI PICSEP workshops are now conducted and sponsored by FOGSI in different member bodies.

Given the low acceptance rate of many journals including ours, you need to be tough enough to accept rejection letters-which will undoubtedly come your way. This is always a depressing occurrence no matter how senior you are, especially if the reviewers have missed the point of your paper. However, sometimes, this is because you have not made the point clearly enough rather than 
because they are biased. You may wish to challenge the rejection and resubmit the manuscript with a detailed rebuttal of the criticisms, but the chances of success are low especially when the covering letter states that they "do not want a resubmission and the decision is final." The urge to immediately send the manuscript to the next journal on your target list is great but do not do this. At least put it to one side for a day or so and then think again about what the reviewers have said and see if it is possible to improve the paper. Most papers can be improved in the light of sensible critical comments.

To improve your skills, help others. Just as a single paper benefits from the cycle of review and revision, the author can benefit from the cycle of careful reading, critiquing others, and writing [11, 12]. By reading critically, you will grow comfortable with principles of organization and coherent argument [13, 14].

\section{References}

1. Hayes JR. A new framework for understanding cognition and affect in writing. In: Levy CM, Ransdell SE, editors. The science of writing: theories, methods, individual differences, and applications. Mahwah: Lawrence Erlbaum;; 1996. p. 1-28.
2. Silvia PJ. How to write a lot. Washington, DC: American Psychological Association; 2007.

3. Hall G, editor. How to write a paper. London: BMJ Books; 1996.

4. Ad Hoc Working Group for Critical Appraisal of the Medical Literature. A proposal for more informative abstracts of clinical studies. Ann Intern Med. 1987;106:598-604.

5. Taddio A, Pain T, Fassos FF, et al. Quality of nonstructured and structured abstracts of original research articles in the BMJ, the CMAJ and the JAMA. Can Med Assoc J. 1994;150:1611-5.

6. Parikh M. Passing the Baton. J Obstet Gynecol India. 2007;57(6):483-4.

7. Hill AB. The reasons for writing. BMJ. 1965;2:870-1.

8. Tufte ER. The visual display of quantitative information. Cheshire: Graphics Press; 1983.

9. The Standards of Reporting Trials Group. A proposal for structured reporting of randomized controlled trials. JAMA. 1994;272:1926-31.

10. Parikh M. Science of writing paper. J Obstet Gynecol India. 2007;57(5):389-90.

11. Huth EJ. How to Write and Publish Papers in the Medical Sciences. 2nd ed. Baltimore: Williams \& Wilkins; 1990.

12. Browner WS. Publishing and Presenting Clinical Research. Baltimore: Lippincott, Williams \& Wilkins; 1999.

13. Devers KJ, Frankel RM. Getting qualitative research published. Educ Health. 2001;14:109-17.

14. Docherty M, Smith R. The case for structuring the discussion of scientific papers. Br Med J. 1999;318:1224-5. 